\title{
A software process for management software systems
}

\author{
Alfs T. Berztiss \\ Department of Computer Science, University of Pittsburgh \\ Pittsburgh,PA 15260,USA \\ (e-mail:alpha@cs.pitt.edu; fax: +412-624-8854)
}

and

SYSLAB, University of Stockholm, Sweden

\begin{abstract}
The business world of today is characterized by very rapidly changing business conditions that require rapid responses. The responses are to be results of decision making, supported by carefully designed management software systems. This paper is a position statement that discusses various factors to be considered in the definition of a software process for developing such systems. Since the rapid change will extend to management software, the software process is to allow rapid configuration of new systems from existing components, and rapid reconfiguration of existing systems. Because the boundary between decision support systems and other classes of business-oriented software is becoming increasingly blurred, we use the term management software systems in preference to decision support systems.
\end{abstract}

\section{Keywords}

Decision support, domain model, management software system, pattern, reuse, software process 


\section{INTRODUCTION}

Kroenke (1992) classifies business software systems into systems for transaction processing, management information, decision support, office automation, and executive support. In full agreement with Alter (1993), who observes that the separation between the DSS, EIS (Executive Information System), and ES (Expert System) worlds is largely artificial, we contend that the distinction between the different types of systems listed by Kroenke has become very blurred, and is in fact disappearing. For example, an order fulfillment system could, in addition to the processing of regular orders, generate periodic summaries for management, use the summaries to assist in decisions of when to increase inventory by initiating manufacturing runs, replace explicit orders as the basis for initiating shipments by automatic monitoring of client inventories, and produce surveys of long-term trends for top management.

Another trend that has to be considered is the very rapid change that characterizes the business environment of today. Daniel Bell's The Coming of the Post-Industrial Society (Bell, 1973) was published as early as 1973. Ten years later George Huber described what a postindustrial organization would look like (Huber, 1984). His basic premise is that postindustrial society will be characterized by accelerating increase in knowledge, complexity, and turbulence, and that postindustrial organizations will therefore have to be qualitatively different from the organizations of the industrial age. The qualitative differences are to be most noticeable in decision making, innovation, and the acquisition and distribution of information. Huber and McDaniel (1986) describe several organizational paradigms, and find that a decision-making paradigm is the most effective for the operation of organizations in the world of today. The main characteristic of this paradigm is the explicit provision of structures and processes that facilitate the making of decisions in the operation of an organization.

The rapid change phenomenon has given rise to the business reengineering (BRE) movement (Davenport, 1993; Hammer and Champy, 1993; Johansson et al, 1993). One aspect of BRE is the recognition of business processes as the defining features of an enterprise. We have discussed this in detail elsewhere (Berztiss, 1995). Another is the need for cooperation within an enterprise and between enterprises at the level of processes. Our order fulfillment system is an example of internal cooperation; a well-known example of inter-company cooperation is the automatic updating by Procter and Gamble of the inventory of its products in Wal-Mart stores (Hammer and Champy, 1993, pp. 60-62).

To summarize, businesses have to cope with rapidly changing business environments. This requires that they assume a decision-making structure, and that decision-making be built into the software systems of an enterprise. These software systems are to support business processes, and a major aspect of their design is to be cooperation, both intra- and interenterprise cooperation. These characteristics have to be taken into account in the design of the development process for decision support software, or, what is a more appropriate term, management software systems. In Section 2 we explore two classes of software development models. Section 3 deals in greater detail with the demands on management software systems, and in Section 4 we consider the software process from the BRE viewpoint. In Section 5 we introduce our software process model. Finally, Section 6 contains some conclusions and recommendations, which have been influenced by our practical experience with patterns. 


\section{MODELS OF THE SOFTWARE DEVELOPMENT PROCESS}

Software process models fall into two major classes. One relates to the capabilities of the organization that develops the software. The other defines the steps that are to be followed in software development, and considers the representations that are to express such a definition of the software process. Let us call the models feature-oriented or managerial, and structureoriented or technical, respectively.

The most comprehensive managerial model is the Capability Maturity Model (CMM), developed at the Software Engineering Institute (SEI) of Carnegie-Mellon University (Humphrey, 1989; Paulk et al, 1993). The CMM puts software developing organizations at five levels, and an organization moves to higher levels by showing that it has mastered all the "key process areas" that define the higher level. Another example is ISO 9001, which is a general quality standard (Paulk, 1995). It identifies minimal requirements for quality control in an organization in the form of 20 clauses. It is related specifically to software by ISO 9000-3. The Bootstrap model (Kuvaja et al, 1994) attempts to combine the SEI and ISO approaches. The CMM is very detailed on how an organization can improve its software development practices, but it is rather rigid, and its use is mainly confined to North America; ISO 9001 is not concerned with detailed steps to improvement. Bootstrap introduces CMM to Europe, but in a flexible form.

A technical model defines steps in the software development process, and recommends an ordering of the steps. Popular technical models have been the waterfall (Royce, 1970) and the spiral (Boehm, 1988) models. The waterfall model assumes a strict ordering of a sequence of steps. The steps could be requirements elicitation, specification, system design, detailed design, coding, testing, and maintenance. However, it has been shown that that these steps must inevitably be intertwined (Swartout and Balzer, 1982). The spiral model allows developers to define their own process steps, and the steps can be revisited over and over again. It can therefore serve as a very general framework for the software process. A generic model, such as the spiral model, has to be transformed into an application-specific software process. Thirteen notations in which the process can be described have been surveyed by Armenise et al (1993). For a detailed description of one of these notations, FUNSOFT, see Deiters and Gruhn (1994).

We have investigated concurrency in a software process model (Berztiss, 1993), and have developed a software process model (Berztiss and Bubenko, 1995) based on the enterprise model introduced by Bubenko et al (1994). The model to be discussed in Section 5 represents an extension of this work. Our model is somewhere between a pure managerial and a pure technical model.

\section{MANAGEMENT SOFTWARE SYSTEMS FOR TODAY AND TOMOR- ROW}

In (Berztiss, 1995) we adapted some guidelines of Huber and McDaniel (1986) that are to facilitate the introduction of a decision-making paradigm into organizations. Here we shall extend and broaden the guidelines, and discuss the significance of the guidelines for the software process, and for the software systems developed by the process. 
Decision-making authority is assigned to appropriate levels in an organizational hierarchy. A three-way partition identifies decisions relating to strategy, operational tactics needed to implement a strategy, and day-to-day operation of an organization. Strategic decisions cannot be generated automatically, but the decision-making process is to be supported by software, which we interpret to cover both programs and information bases. Tactical decision making can be partially automated, and the decisions that arise in day-to-day operation can be automated for the most part.

Decision makers must have the specialized knowledge appropriate to their level of decision making. This requires not only specialized skills to interpret information, but userfriendly access to all required information, and to software tools that are to support interpretation of the information and the actual decision making.

Situations are identified as standard and nonstandard. This refers to situations that arise in day-to-day operation of an organization. Decision making with respect to standard situations can be standardized and automated. Nonstandard situations require initiative and flexibility. This implies that management software systems must be constantly monitored by people, and that a switch to manual control of a situation can be effected seemlessly. In particular, messages flowing through an organization relate to standard and nonstandard situations. Response to "standard" messages is to be automated; "nonstandard" messages are to be rapidly chanelled to appropriate decision making personnel. A nonstandard message may affect strategy.

Decision making is managed. Under the decision-making paradigm the making of decisions is the central activity in an organization, and it cannot be left to chance. What this means is that all business processes are to be explicitly defined, and all routine decision making is to be supported by appropriate management software systems. Moreover, an organization should be able to switch into an exceptional mode of operation when truly exceptional situations arise, and this without throwing anybody into panic.

Processes are "owned". Business reengineering puts much emphasis on business processes, and it has been recommended that a process and the data that give primary support to the process should both be "owned" by the same manager - see, e.g., (Davenport, 1993, p.88). This implies that our current views on data bases, and a preference for their centralized management have to be reconsidered. Moreover, a process can then be regarded as being supported by an autonomous management software system that cooperates with software systems that support other business processes, all having the common aim of advancing the objectives of an enterprise.

Cooperation is emphasized, both within an organization and between organizations. This was briefly touched upon in Section 1 . Cooperation within an organization relates to all three kinds of decision making, and is to be supported by appropriate tools that facilitate group decision making. Cooperation between organizations has related for the most part to day-today operation, but we can expect that in the future more attention will be given to softwaresupported strategy formulation in the setting up of alliances of companies and their suppliers - for a general discussion see (Lewis, 1995). Cooperation by means of software systems requires that they be properly coordinated - Malone and Crowston (1994) survey various aspects of coordination.

Intelligent agents are in use. When decision making by software reaches an advanced level, e.g., when a software system controls the investment portfolio of an enterprise, it becomes appropriate to talk of intelligent agents. However, there is wide disagreement on when exactly a piece of software becomes an agent - apart from the inclusion of a learning 
component in an agent, there is little to distinguish agents from expert systems. Although practical successes have been few as yet (Greif, 1994), learning components in management software systems are to be given greater attention. Agents can assume particular significance in the context of cooperative management software systems, where they are to cooperate, and are to facilitate cooperation.

Domain knowledge becomes part of software. We consider the incorporation of domain knowledge into management software systems as perhaps the most important of the trends considered in this section. Our interest is in domain knowledge, domain models, and domain analysis. Domain knowledge is the entire corpus of data, rules, and processes that characterizes a discipline. Some of it is codified in standards and handbooks, but most of it is distributed in the collective memory of practitioners of the discipline. A domain model is an abstraction that consists of only those parts of the domain knowledge that are relevant to a particular application. The purpose of domain analysis is to develop domain models. We classify domain models into occupation models, enterprise models, process models, and situation models. An occupation model captures the knowledge that relates to the practices followed by members of an occupation as an explicit structure. For the most part this is expressed in the form of business rules. An enterprise model provides insight into the objectives of an enterprise, i.e., the goals that a management software system is to assist in achieving. It also shows the management structure of the enterprise. The ownership of processes and their data ultimately depends on this structure. Process models are easiest to define: they capture the essence of business processes. A situation model relates to a specific context. An interesting aspect of situation analysis relates to exceptions. For example, overbooking can be incorporated into some reservation systems, with the degree of overbooking for a given setting determined from domain knowledge, which is to include a consideration of enterprise objectives. An exception arises when, due to overbooking, actual demand exceeds availability by an unexpectedly large margin.

\section{REENGINEERING AND THE SOFTWARE PROCESS}

In (Berztiss, 1995) we define BRE in terms of sixteen steps. We shall now use these reengineering steps as a framework for the definition of software development processes, and relate the reengineering steps to a process defined within the framework, and to the software systems to be developed under this process.

1. Management commitment. There has to be full management support for the introduction of a process-oriented approach to software development. In addition, management has to insist that each software project is well planned, subjected to a thorough cost-benefit study, and is smoothly integrated into the organizational structure. This necessitates some understanding of the software process by top management.

2. Manager selection. Most organizations now have a CIO (Chief Information Officer), and this executive officer must see to it that top management understands the ultimate benefits to be derived from an improved software process, i.e., a decision-making process that requires project planning and cost-benefit analysis. An operational manager is to supervise an initial overhaul of the operation of the IS (Information Systems) department, to bring it in line with modern practices as defined by the managerial process models of Section 2 , and the introduction of incremental improvements after the initial overhaul. 
3. Reengineering teams. The overhaul of information systems development is a typical BRE task, to be tackled exactly like any other BRE task. In particular, basing the software development process on the use of self-managing development teams is to be encouraged.

4. Initial education. The IS personnel must have full understanding of the software process, both under the feature- and the structure-oriented forms. This is essential for obtaining personnel support for the process. One task of education is to make clear that no spectacular improvement can be expected in the short term, but that long-term improvement will be achieved if software development follows a well-defined process.

5. Process identification. We have to distinguish between the software development process, and software systems that this process is to produce. In this step a generic software process is to be defined under consideration of both features and structure.

6. Process specification. This step relates to software systems, but an essential property of a software system is that it defines a process. There has to exist a precise requirements document for each software system. Preferably this should be a formal specification document.

7. Development of alternative implementation plans for each process. Although under Steps 5 and 6 we referred to software systems, our real concern is with total systems in which tasks are allocated to hardware, software, and people. At this stage different allocation schemes can be proposed, and an implementation plan developed for each scheme. Note that the software process itself can be implemented in various ways, and the various alternatives should be defined as part of this step.

8. Cost-benefit study for each plan and selection of an appropriate plan. Each of the schemes of Step 7 is now subjected to cost-benefit analysis. Moreover, the software components of these plans do not have to be implemented under the same software process, i.e., different specific processes can be derived from the generic process of Step 5, as noted under Step 7. Each relevant combination of software system and software process is to be considered in the cost-benefit analysis.

9. Infrastructure definition based on the selected plan. This relates primarily to the software process, and the infrastructure consists of CASE (Computer Assisted Software Engineering) tools, and groupware that is to allow IS personnel to work in collaboration. This in turn requires that there be adequate network facilities to support the groupware. The infrastructure requirements do not depend on individual software projects - they will be determined by the mix of the software systems that an organization develops.

10. Setting of priorities for implementation of processes and infrastructure. Here we have to consider two aspects. As explained above, software process and infrastructure are related, and the priorities in infrastructure upgrading will be determined by a cost-benefit analysis that will again depend on the product mix. Further, no organization possesses the resources to implement all the software systems it can use, so a cost-benefit analysis is to define a priority schedule. However, it has to be realized that cost-benefit analysis in this context is still very subjective, and will remain so until we have improved our understanding of the benefits that are to be derived from specific software systems.

11. Second education phase. This is an on-going effort, relating both to the software process and to the software systems. As our understanding of the effectiveness of different aspects of the software process grows, this understanding has to be conveyed to IS personnel. As regards software systems, one of the major problems is inadequate understanding of application domains (Curtis et al, 1988). There have to be institutionalized procedures for making the relevant IS personnel familiar with the domains for which they are developing software systems. 
12. Pilot implementation. Just as there has to be a priority list for the implementation of software systems (Step 10), so there has to be a priority list for the components of a software process model. The taking of measurements that relate to the software process and to the software systems produced by the process is to begin at once, but the effective utilization of the measurements can only begin when there are enough of them, and this takes time. The first priority, then, is emphasis on measurements. At the same time, all other features that define a mature software process in accordance with a managerial process model are to be examined, and the sequence for their introduction is to be determined by the "organizational culture" of the IS department, i.e., by how ready IS is to accept responsibility for each feature.

13. Reexamination of the BRE effort. The activities of Steps 10 through 12 should not be considered in isolation. They depend on each other and reinforce each other, and an early demonstration of the effectiveness of this consolidated approach to the reengineering of IS will determine the willingness of top management to allocate additional resources to a continuation of the reengineering of IS.

14. Completion of implementation. The ultimate aim of any IS department should be to have an excellent software process in place, but, for example, to reach the higher levels on the SEI process maturity scale takes time, resources, and unreserved management support. In Step 13 it was essential to show that resources will be well spent; in Step 14 it is essential to spend the resources well.

15. Continuing automation of processes. Again we have to differentiate between the software process and software systems. At Level 5 on the SEI scale, the software process is to optimize itself. This will remain a manual activity, carried out by people supervising the process. However, reengineering of IS improves productivity and the skills of IS personnel. This allows software systems to become more sophisticated.

16. Automatic linkages to the environment. The environment of the IS department is the entire organization of which it is a part, and the software systems developed by IS should assist in a coordinated operation of the organization. One aim of BRE should be the integration of all activities of an organization - this depends greatly on how well the software systems that support these activities are integrated. A broader environment includes entities with which an organization cooperates, such as suppliers, government agencies, and trade groups.

\section{A SOFTWARE PROCESS MODEL}

Rapid construction of management software systems requires a well-defined process. In the preceding three sections we examined requirements for this software process from three viewpoints. In Section 2 a distinction was made between managerial and technical models. In Section 3 we examined some current trends relating to the operation of organizations. A most important trend is BRE, and we took a BRE approach to the software process in Section 3. All these trends and factors are to have an effect on the process for developing management software systems. However, to give full justice to this effect is beyond the scope of a conference paper, and here we merely state that the process model introduced below was developed under consideration of the material of Sections 2-4. Moreover, the process model can be presented in only a very sketchy form.

In (Berztiss and Bubenko, 1995) we introduced a software system development model, which, modified and extended, is shown here as Figure 1. In this model, system development 
begins with objectives and ends with a management software system. The objectives determine a domain model that consists of three basic components: a structured information base, procedures for changing the information base (events), and processes. This somewhat informal model is converted into a formal functional specification. There are also nonfunctional aspects of a software system, such as usability, which can rarely be stated in formal terms they are represented by the "Nonfunctional requirements" box. This development process is defined in terms of various concepts, and different roles in the process are allocated to actors, which can be people or software tools. Several versions of the system can be generated, and, during the lifetime of a particular version, it is represented in different forms, such as requirements diagrams, specification, and code. There must always be a clear understanding of which version a particular representation belongs to (version control), and that all representations of a version are consistent (configuration control).

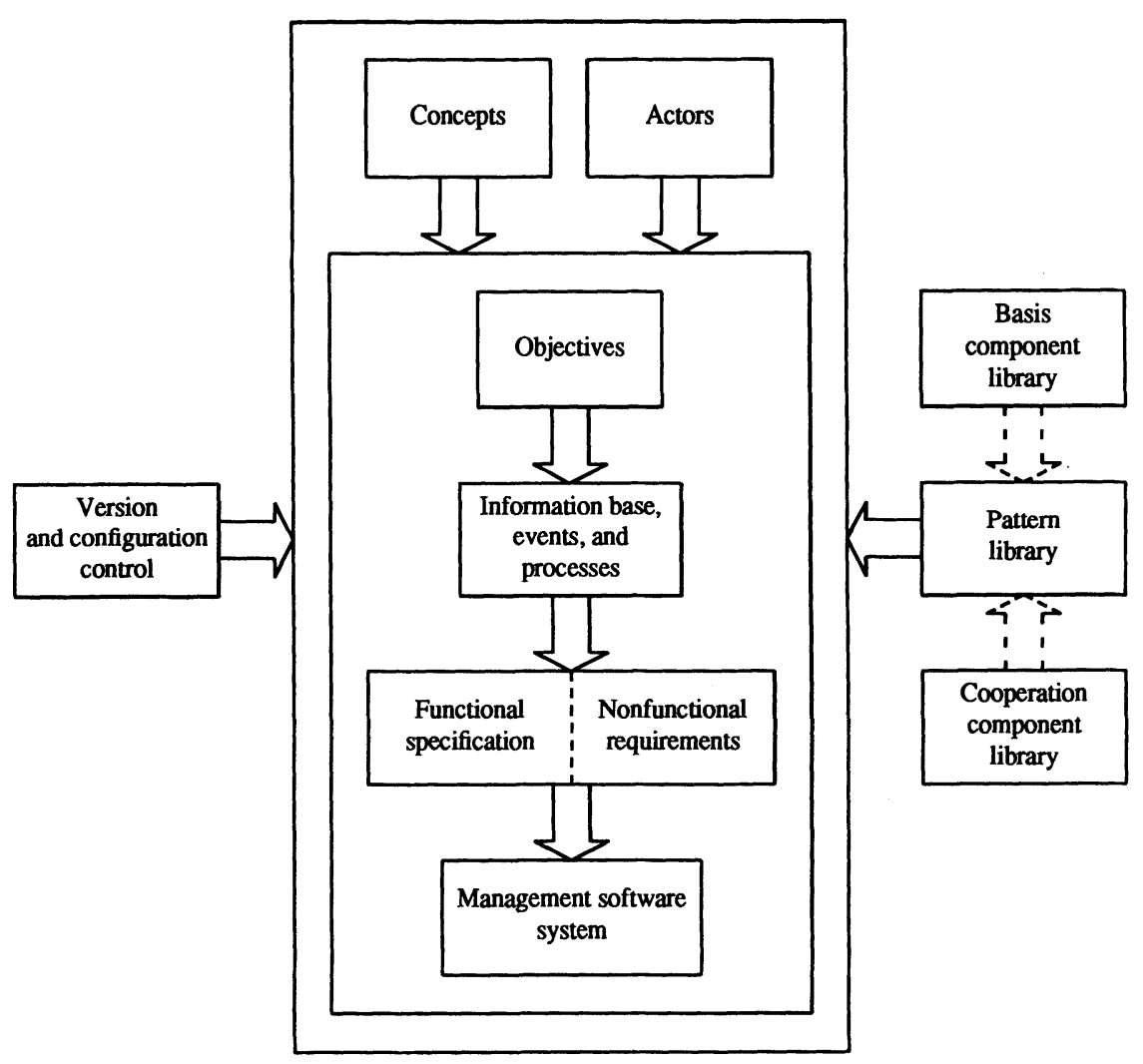

Figure 1 A software process model for management systems 
The more enclosed a box, the more specific is its role in the process. The box for version and configuration control, and the three boxes for the libraries are not enclosed, which means that they are not influenced by what particular software product is being developed, i.e., they are fully application independent.

The main change in this model from the Berztiss and Bubenko (1995) model is the addition of the libraries. Lately some domain knowledge has been expressed as patterns - see, e.g., (Coplien and Schmidt, 1995). We interpret a pattern to be a generic template that defines the structure of a problem and its solution, but that has to be refined to become applicable in a specific situation. We can relate the four domain models of Section 3 to patterns. The occupation and enterprise models suggest what management software systems are needed and what patterns are relevant in the setting up of the systems. A process model imposes structure on a pattern, and a situation model relates a process to the setting in which it is to operate - in particular, exceptional conditions in the operation of a process are covered by the situation model. In Figure 1 we have a basis component library and a cooperation component library. These components are gathered up into a pattern.

Let us take a concrete example, a rental car company. A look at its operation allows us to identify a set of very general patterns that are constructed almost exclusively from "basis components". These patterns relate to reservations, actual rentals, acquisition and disposal of inventory, tracking of transaction trends, management of distributed sites, etc. The reservations and rental patterns arise in the hotel, airline, car rental, and formal wear rental businesses. This is by no means an exhaustive list - Maiden and Sutcliffe (1992) point out the similarity between a theatre reservation system and a university course registration system; we note that both are instances of the reservation pattern. Tracking of transaction trends should be practiced by every enterprise - a downward trend is a danger signal; an upward trend of car rentals should trigger inventory build-up; an upward trend in hotel occupancy rates can justify an increase in room charges. Management of distributed sites is particular to car rental: a car may be picked up and dropped off at different sites, and transaction trends at different sites may suggest a redistribution of cars.

An example of pattern constructed primarily from "cooperation components" is the scheduling of a meeting, which can involve personnel from several enterprises. This can be handled by an intelligent agent. The pattern requires the agent to consult intended participants regarding their availability, and negotiate a mutually acceptable time slot, which can be complicated if the participants' calendars show no common available time slot. Depending on the width of the negotiated time window, the agent has to decide whether to hold a faceto-face or an electronic meeting. In the worst case, there can be a switch to a group decision mode in which participants cooperate asynchronously via a blackboard structure. The selection of the blackboard structure is a decision relating to software architectures - see, e.g., (Shaw, 1995).

The actors box has a twofold significance. First, it has to be decided which parts of the software process are to be carried out by people and which by tools. Maiden and Sutcliffe (1993) advance strong arguments that reuse is to be very much people-oriented. In terms of Figure 1, the four boxes relating to objectives, the three-component requirements, patterns, and functional specifications define a difficult system construction task, and only people are capable of effectively carrying out this task.

Second, in the system that is being developed, various tasks are to be allocated to people, software, and hardware. Considering people and software alone, the allocation relates to routine tasks, routine exceptions, and unexpected exceptions. With regard to order fulfillment, 
this is a routine process when there are no shortages. Routine exceptions arise when there are inventory shortages, and an unexpected exception arises when the warehouse staff go out on strike. The first two cases can be handled by software, the last, because it is unexpected, has to be handled by people, but in cooperation with the software system.

\section{CONCLUSIONS AND RECOMMENDATIONS}

The two most important tasks for the future are the setting up of a pattern library, and the development of a "pattern selection apprentice". We have started work on the former, and in two directions - to prove that the approach is quite general. One direction is in computer science, and is concerned with patterns relating to safety-critical situations (Berztiss, 1996); the other relates to management software, and consists of patterns suggested by the analysis of the operation of a rental car business, which we referred to earlier. Some of the rental business patterns deal with the gathering of information that is to support decision making, and the actual making of decisions. For example, an analysis of rental data can suggest a redistribution of rental objects over the locations at which the enterprise operates, and an analysis of repair and resale data will suggest when a rental object is to be taken out of service. These generic patterns are as easily adaptable to rental videos as to rental cars. The rental data pattern can also indicate shortages, which in the case of rental cars may lead to purchase of additional cars, but in the case of a hotel to raised room rates.

The base of our pattern library consists of the generic patterns, which are merely capsule outlines in natural language. A pattern for a specific application has also a natural language version that consists of five entries. They are (1) Triggered by, which establishes what it is that initiates the activities grouped under the pattern; (2) Activities, which gives an outline of all activities that are part of the pattern, an indication of the conditions under which each activity takes place, and an indication of how the activities are related to each other; (3) Information base changes, which indicates those parts of the information base supporting the carrental process that are to be affected by the activities of the pattern; (4) Affects, which identifies all patterns that can be affected by this pattern, and states the conditions under which this pattern would interact with other patterns; (5) Notes, which can contain any information deemed relevant by the author of the pattern, e.g., an explicit indication of what is not considered in the pattern. These patterns are next defined in the specification language SF (Berztiss, 1990) is currently the best introduction to SF.

With the capsule summaries to refer to, the setting up of patterns for a reservation and the cancellation of a reservation in the rental-car context took about 25 minutes for initial design and about 10 minutes for review on the following day. These patterns were adapted for the reservation and cancellation of a restaurant table in approximately six minutes. Being given just the capsule summaries, teams of rather inexperienced undergraduate students in one of the author's courses have implemented and fairly thoroughly tested an information system for a rental-car operation in around 360 person-hours (a team consisted of six students, and each student contributed approximately 60 hours to the project).

The pattern selection apprentice is to be a specialization of the software apprentices described by Rich and Waters (1990). By consulting the requirements for a management software system and a pattern library, the apprentice is to decide which patterns are applicable in a given situation. Initially the apprentice is merely to help software developers 
navigate through a library of patterns.

The ultimate purpose of a management software system is to exercise control. We distinguish between three kinds of software: a conventional program that is fully deterministic and can exercise control over a process or part of a process entirely on its own; an ES that also exercises total control, but that may be under continuous modification as the understanding of the process continues to improve over time; a DSS that assists a human decision maker, but does not make control decisions entirely on its own. Thus, a fully understood process is controlled by a conventional program, a process that is reasonably well understood and about which our understanding continues to improve is under control of an ES, and a process that is not so well understood is under partial control of a DSS. If our understanding is so poor that we cannot express our knowledge about the process as software, then we have to depend entirely on human versatility in dealing with it. How far a management system can be advanced along this progression from full human control to a decision support system to an expert system to a conventional program depends on how well a control situation is understood, and how well it can be explicitly described. This applies both to the software process, and to the systems with which the software process is to deal.

\section{REFERENCES}

Alter, S. (1993) Why persist with DSS when the real issue is improving decision making? In Decision Support Systems: Experiences and Expectations (eds. T. Jelassi, M.R. Klein, and W.M. Mayon-White), 1-11, North-Holland.

Armenise, P., Bandinelli, S., Ghezzi, C., and Morzenti, A. (1993) A survey and assessment of software process representation formalisms. Int. J. Software Eng. Knowledge Eng. 3, 401-426.

Bell, D. (1973) The Coming of the Post-Industrial Society. Basic Books.

Berztiss, A. (1990) Formal specification methods and visualization. In Principles of Visual Programming Systems (ed. S.-K. Chang), 231-290, Prentice Hall.

Berztiss, A. (1993) Concurrent engineering of information systems. In Proc. IFIP WG8.1 Working Conf. on Information System Development Processes (eds. N. Prakash, C. Rolland, B. Pernici), 311-324, North-Holland.

Berztiss, A.T. (1995) Software Methods for Business Reengineering. Springer-Verlag.

Berztiss, A.T. (1996) Unforeseen hazard conditions and software cliches. To appear in High Integrity Systems 1.

Berztiss, A.T., and Bubenko, J.A. (1995) A software process model for business reengineering. In Information Systems Development for Decentralized Organizations, 184-200, Chapman \& Hall.

Boehm, B.W. (1988) A spiral model of software development and enhancement. Computer 21, (5), 61-72.

Bubenko, J.A., Rolland, C., Loucopoulos, P., and DeAntonellis, V. (1994) Facilitating "fuzzy to formal" requirements modelling. In Proc. IEEE Internat. Conf. on Requirements Eng.

Coplien, J.O., and Schmidt, D.C, eds. (1995) Pattern Languages of Program Design. Addison-Wesley.

Curtis, B., Krasner, H., and Iscoe, N. (1988) A field study of the software design process for large systems. Communications of the ACM, 31, 1268-1287. 
Davenport, T. H. (1993) Process Innovation: Reengineering Work through Information Technology. Harvard Business School Press.

Deiters, W., and Gruhn, V. (1994) The FUNSOFT net approach to software process management. Int. J. Software Eng. Knowledge Eng. 4, 229-256.

Greif, I. (1994) Desktop agents in group-enabled products. Comm. ACM 37, (7), 100-105.

Hammer, M., and Champy, J. (1993) Reengineering the Corporation: A Manifesto for Business Revolution. Harper Business.

Huber, G.P. (1984) The nature and design of post-industrial organizations. Management Science 30, 928-951.

Huber, G.P., and McDaniel, R.R. (1986) The decision-making paradigm of organizational design. Management Science 32, 572-589.

Humphrey, W.S. (1989) Managing the Software Process. Addison-Wesley.

Johansson, H. J., McHugh, P., Pendlebury, A. J., and Wheeler, W. A. (1993) Business Process Reengineering: BreakPoint Strategies for Reengineering. Wiley.

Kroenke, D. (1992) Management Information Systems. McGraw-Hill.

Kuvaja, P., Simila, J., Krzanik, L., Bicego, A., Saukkonen, S., and Koch, G. (1994) Software Process Assessment and Improvement - The BOOTSTRAP Approach. Blackwell Business.

Lewis, J.D. (1995) The Connected Corporation. Free Press.

Maiden, N.A., and Sutcliffe, A.G. (1992) Exploiting reusable specifications through analogy. Comm. ACM 35, (4), 55-64.

Maiden, N.A.M., and Sutcliffe, A.G. (1993) People-oriented software reuse: the very thought. In Advances in Software Reuse (eds. R. Prieto-Diaz and W.B. Frakes), 176-185, IEEE Computer Society Press.

Malone, T.W., and Crowston, K. (1994) The interdisciplinary study of coordination. ACM Computing Surv. 26, 87-119.

Paulk, M.C. (1995) How ISO 9001 compares with the CMM. IEEE Software 10, (4), 74-83.

Paulk, M.C., Weber, C., Garcia, S., Chrissis, M.B., and Bush, M. (1993) Key practices of the Capability Maturity Model Version 1.1. SEI Report CMU/SEI-93-TR-25, Software Engineering Institute of Carnegie-Mellon University.

Rich, C., and Waters, R.C. (1990) The Programmer's Apprentice. ACM Press, 1990.

Royce, W.W. (1970) Managing the development of large software systems. In Proc. IEEE WESCON, 1-9.

Shaw, M. (1995) Architectural issues in software reuse: it's not just functionality, it's the packaging. In Proc. ACM SIGSOFT Symp. Software Reusability (special issue of ACM SIGSOFT Software Engineering Notes), 3-6.

Swartout, W., and Balzer, R. (1982) On the inevitable intertwining of specification and implementation. Comm. ACM 25, 438-440. 


\section{BIOGRAPHY}

Prof. Berztiss received the $\mathrm{PhD}$ in Theoretical Physics from the University of Melbourne in Australia. Since 1970 he has been with the Computer Science Department of the University of Pittsburgh. Since 1984 he has also been a research associate of SYSLAB of the University of Stockholm. Dr. Berztiss is a Fellow of the Australian Computer Society, and belongs to ACM, IEEE, European Association for Theoretical Computer Science, and IFIP Working Groups 8.1 (Design and Evaluation of Information Systems) and 8.3 (Decision Support Systems). He has published three books and numerous technical papers. He has been a consultant with UNIDO, SEI, IBM, etc. His research interests include conceptual modeling in the reengineering of organizations, specification and prototyping of software, reliability engineering of software, treatment of uncertain data, and curriculum development for software engineering education. 